\title{
Variant Domination Types for a Complete h-ary Tree
}

\author{
Manal N. Al-Harere \\ Rasha Jalal Mitlif* \\ Fatema Ahmad Sadiq
}

Department of Applied Sciences, Branch of Mathematics and Computer Applications, University of Technology, Baghdad, Iraq.

"Corresponding author: 100035@uotechnology.edu.iq, 10096@uotechnology.edu.iq ${ }^{*}, 10122 @$ uotechnology.edu.iq

*ORCID ID: https://orcid.org/0000-0003-1125-4439, https://orcid.org/0000-0001-5033-8753*, https://orcid.org/0000$\underline{0001-5159-1574}$

\author{
Received 30/5/2019, Accepted 20/10/2020, Published 30/3/2021
}

\begin{abstract}
Graph $G=(V, E)$ is a tool that can be used to simplify and solve network problems. Domination is a typical network problem that graph theory is well suited for. A subset of nodes in any network is called dominating if every node is contained in this subset, or is connected to a node in it via an edge. Because of the importance of domination in different areas, variant types of domination have been introduced according to the purpose they are used for. In this paper, two domination parameters the first is the restrained and the second is secure domination have been chosn. The secure domination, and some types of restrained domination in one type of trees is called complete $h-\operatorname{ary} \operatorname{tree}\left(T_{c, h, r}\right)$ are determined.
\end{abstract}

Keywords: Complete $h$-ary trees, Domination, Restrained domination, Secure domination Mathematical subject classification: 05C69

\section{Introduction:}

Let $G=(V, E)$ be a finite undirected simple graph with vertex set $V=V(G)$ and edge set $E=E(G) . N(v)=\{u \in V: u v \in E(G)\}$ and $N[v]=N(v) \cup\{v\}$, are open neighborhood and closed neighborhood sets of $v$ respectively. The induced subgraph $G[D]$ is a graph of vertices set $D \subseteq V(G)$ of a graph $G$ together with any edges whose endpoints are both in this subset. Any notion or definition which is not found here could be found in $(1,2)$. An independent vertex set of a graph $G$ is a subset of the vertices such that no two vertices in the subset represent an edge of $G$, (2).

In any graph $G$ the set $D \subseteq V(G)$ is a dominating set if every vertex $v \in V$ is either an element of $D$ or is adjacent to an element of $D$. The domination number of $G$, denoted by $\gamma(G)$, is the cardinality of a minimum dominating set of $G$, (3). Because of the importance of domination in different areas, variant types of domination have been introduced according to the purpose they are used for. The domination parameters have been formed either by putting a condition on the vertices of a dominating set $D$, or by putting a condition on the vertices in $V-D$ or on both. A total dominating set $D$ of a graph $G$ is a dominating such that $G[D]$ has no isolated vertices.st. An independent dominating set is a vertex subset which is both independent and dominating.

Various types of domination of a graph $G$ have been defined and studied by several authors, they are listed in the appendix of Haynes $(4,5)$. For more details about parameters of domination number that depend on vertex dominating with condition on dominating set $D$, see $(6,7,8,9)$, and for condition on vertices in set $V-D$, see $(10,11,12,13,14)$.

Here, variation types of the domination theme, namely that of restrained domination are studied. In a graph $G$ with a dominating set $D$, and the following condition put on the vertices of set $V-D$, such that the open neighborhood of every vertex in $G[V-D]$ is not an empty set. If this condition is verified then $D$ is a restrained dominating set in $G$. The number $\gamma_{r}(G)$, is the minimum cardinality of a restrained dominating set of $G,(15)$. When, $D$ is a restrained and independent then it is called an independent restrained dominating set, (16). The independent restrained domination number of $G$, denoted by $\gamma_{i r}(G)$, is the smallest cardinality of an independent restrained dominating set of $G$. 
The total restrained domination number of $G$, denoted by $\gamma_{t r}(G)$, is the smallest cardinality of a total restrained dominating set of $G$ (when the dominating set $D$ is restrained and has no isolated vertices in $G[D]),(17)$. For the following condition: each $u \in V-D$, there exists a vertex $v \in D$ such that $u v \in E$ and $(D-\{v\}) \cup\{u\}$ is a dominating set, if the this condition is verified then $D$ is a secure dominating set of $G$.The minimum cardinality of a secure dominating set in $G$ is the secure domination number denoted by $\gamma_{S}(G)$, $(18,19)$.

In this paper, the domination in one type of trees is studied. Let $h \geq 1, r \geq 0$ be integers. The complete $\mathrm{h}-$ ary tree of depth $r$, denoted by $\left(T_{c, h, r}\right)$, is a complete $(c)$ rooted tree in which every non-pendent vertex has exactly $h$ children, and the distance from the root to each pendent is exactly $r$. The root vertex $\mathrm{h}$ been labeled by $v_{0}$, (as an example, see Fig. 1).

Here, various types of dominating parameter (restrained domination, total restrained domination, independence restrained domination, and secure domination) number of a complete $h$-ary root, $h \geq$ $2, r \geq 3$ are determined.

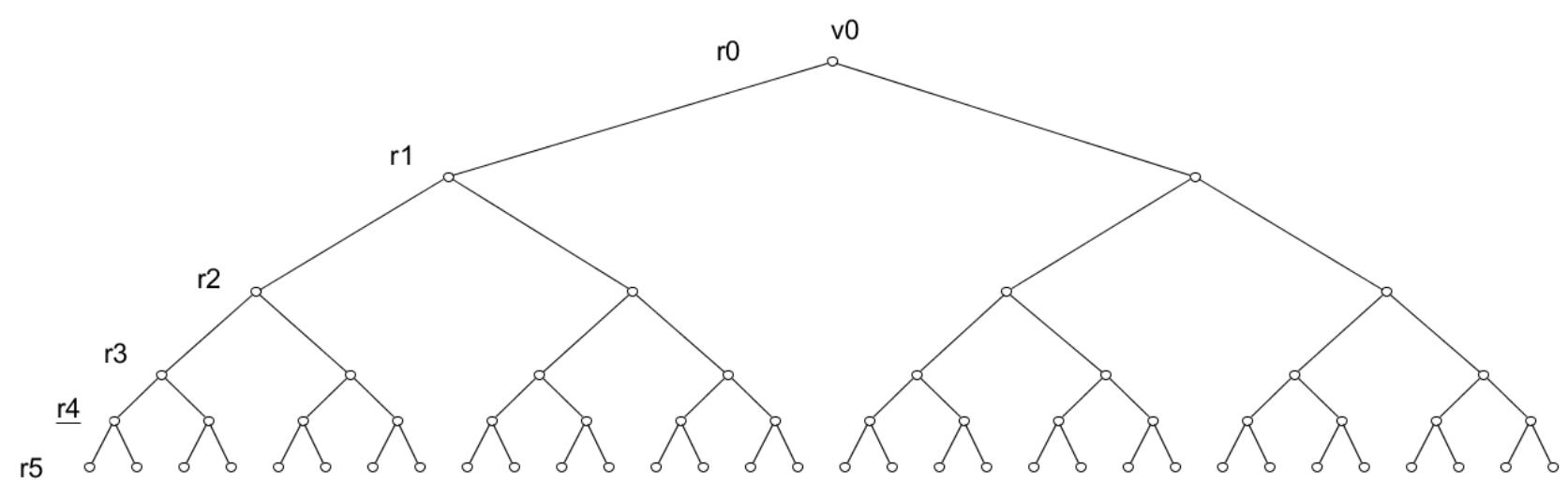

Figure 1. $T_{c, 2,5}$

Lemma 1 (15) If $D$ is a minimum restrained dominating set of a tree $T$; then every pendent of $T$ belongs to $D$.

\section{Restrained domination in a complete $\boldsymbol{h}$-ary tree}

In this section, restrained, independent restrained and total restrained domination for a complete $h$-ary tree $T_{c, h, r}$, are determined:

Theorem 2 If $G=T_{c, h, r}$, is a complete h-ary tree; then for $r \geq 3$,

$$
\gamma_{r}(G)=\left\{\begin{array}{l}
\frac{h^{r+3}-1}{h^{3}-1} \text { if } r \equiv 0(\bmod 3), \\
{\left[\frac{h^{r+3}}{h^{3}-1}\right] \text { if } r \equiv 1(\bmod 3),} \\
{\left[\frac{\left.h^{r+3}\right]}{h^{3}-1}\right]+h \text { if } r \equiv 2(\bmod 3) .}
\end{array}\right.
$$

\section{Proof.}

Looking for a set such that, this set contains as possible a minimum restrained dominating set. So, let's consider $\quad D=\cup_{i=0}^{\left(\frac{r}{3}\right)} D_{i}$, where $D_{i}=$ $\{v: v$ is a vertex of depth $r-$ 3i in $G$ \} for all $\mathrm{i}=0,1, \ldots,\left\lfloor\frac{r}{3}\right\rfloor \quad$ and $\quad|D|=$ $\sum_{i=0}^{\left.\mid \frac{r}{3}\right\rfloor} h^{r-3 i}$. Three cases are obtained.

(i) If $r \equiv 0(\bmod 3)$, then set $D$ dominates the vertices of $G$ where, induce subgraph $G[V-D]$ has no isolated vertices, since between any two sets $D_{i}$ and $D_{i+1}, i=0,1, \ldots, \frac{r}{3}-1$, there are adjacent vertices of depths $r-3 i-1$ and $r-3 i-$ $2, i=0,1, \ldots, \frac{r}{3}-1$. Therefore, in this case set $D$ is a restrained dominating set in $G$. Thus, $\gamma_{r}(G) \leq$ $\sum_{i=0}^{\frac{r}{3}} h^{r-3 i}$. If there is a dominating set $A$ of $|A|$ vertices with $|A|<\sum_{i=0}^{\left\lfloor\frac{r}{3}\right\rfloor} h^{r-3 i}$ then $A$ will definitely not contain the pendant vertices and according to Lemma 1.1, every leaf (pendant) of $T$ belong to the minimum restrained dominating set in $T$, then $A$ cannot be restrained. Thus, set $D$ is the minimum set. So that in this case $\gamma_{r}(G)$ equals to $\gamma_{r}(G)=|D|=\sum_{i=0}^{\frac{r}{3}} h^{r-3 i}=\frac{\left(h^{r+3}\right)-1}{h^{3}-1}$

(ii) If $r \equiv 1(\bmod 3)$, then the vertices which are not dominated by the set $D$ is only the root vertex, so $D \cup\left\{v_{0}\right\}$ is the minimum restrained dominating set in $G$, (as an example, see Fig. 2).

Therefore in this case, 


$$
\begin{gathered}
\gamma_{r}(G)=1+\sum_{i=0}^{\frac{r-1}{3}} h^{r-3 i}=1+\frac{\left(h^{r+3}-h\right)}{h^{3}-1} \\
=\left\lceil\frac{h^{r+3}}{h^{3}-1}\right\rceil
\end{gathered}
$$

(iii) If $r \equiv 2(\bmod 3)$, then the set $D$ can dominate the graph as a minimum restrained domination except the vertices of depth one plus the root vertex $v_{0}$.

$.1+$ Thus $\gamma_{r}(G)=1+h+\sum_{i=0}^{\left\lfloor\frac{r}{3}\right\rfloor} h^{r-3 i}=\left\lceil\frac{h^{r+3}}{h^{3}-1}\right\rceil$

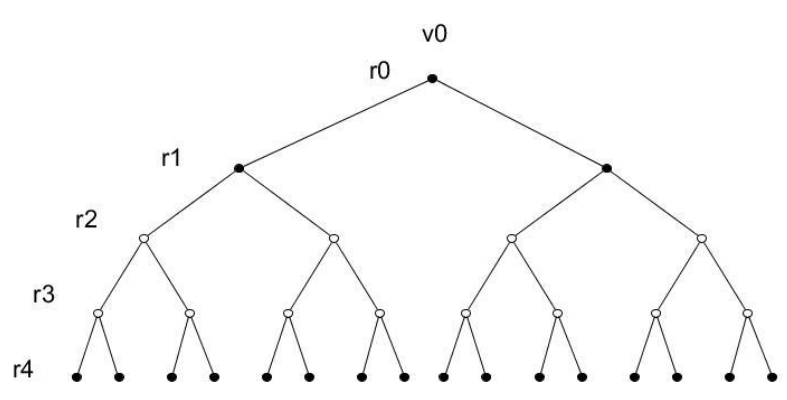

Figure 2: Restrained dominating set in $T_{c, 2,4}$

Theorem 3 If $G=T_{c, h, r}$ is a complete h-ary tree; then $\gamma_{i r}(G)=\gamma_{r}(G)$ for $r \equiv 0(\bmod 3)$.

\section{Proof.}

Looking for a set such that, this set contains as possible a minimum independence restrained dominating set. So, let's consider $D=\mathrm{U}_{j=0}^{\left\lfloor\frac{r}{3}\right\rfloor} D^{j}$, where $\quad D^{j}=\{v: v$ is a vertex of depth $r-$ $3 j$ in $G\}$ and $|D|=\sum_{j=0}^{\left.\mid \frac{r}{3}\right\rfloor} h^{r-3 j}$, so there are two cases:

(i) if $r \equiv 0(\bmod 3)$, then $|D|=\gamma_{r}(G)=$ $\gamma_{i r}(G)=\sum_{j=0}^{\frac{r}{3}} h^{r-3 j}$.

(ii) if $r \equiv 1,2(\bmod 3)$, the graph has no independence restrained dominating set.

Theorem 4 If $G=T_{c, h, r}$ is a complete $h$-ary tree, then for $r \geq 5$,

$$
=\left\{\begin{array}{cc}
(h+1) \frac{h^{r+3}-1}{h^{4}-1}, & \text { if } r \equiv 1(\bmod 4), \\
1+\left(h^{2}+h\right)\left(\frac{h^{r+2}-1}{h^{4}-1}\right), & \text { if } r \equiv 2(\bmod 4), \\
1+\left(h^{3}+h^{2}\right)\left(\frac{h^{r+1}-1}{h^{4}-1}\right), & \text { if } r \equiv 3(\bmod 4), \\
1+3 h+\left(h^{4}+h^{3}\right)\left(\frac{h^{r}-1}{h^{4}-1}\right), & \text { if } r \equiv 0(\bmod 4) .
\end{array}\right.
$$

\section{Proof.}

Looking for a set such that, this set contains as possible a minimum total restrained dominating set. So let's consider $D=\cup_{i=0}^{\left\lfloor\frac{r-1}{4}\right\rfloor} D_{i}$, where $D_{i}=$ $\{v: v$ is a vertex of depth $r-4 i$ and $r-4 i-$ 1 in $G\}$ and $|D|=\sum_{i=0}^{\left\lfloor\frac{r-1}{4}\right\rfloor}\left(h^{r-4 i}+h^{r-4 i-1}\right)$, so there are four cases:

(i) if $r \equiv 1(\bmod 4)$, then the vertices in set $D$ dominate $G$ and $G[V-D]$ is a graph with no isolated vertices, since between any two sets $D_{i}$ and $D_{i+1}, i=0,1, \ldots, \frac{r-1}{4}-1$, there are adjacent vertices of depths $r-4 i-2$ and $r-4 i-$ $3, i=0,1, \ldots, \frac{r-1}{4}-1$. Therefore, $D$ is the restrained dominating set in $G$.

Thus $\gamma_{r}(G) \leq \sum_{i=0}^{\frac{r-1}{4}}\left(h^{r-4 i}+h^{r-4 i-1}\right)$. , (as an example, see Fig. 3).

Assuming there exists a set $A$ such that this set is a dominating set in $G$ with $|A|<\sum_{i=0}^{\frac{r-1}{4}}\left(h^{r-4 i}+\right.$ $h^{r-4 i-1}$ ), then set $A$ will definitely not contain the pendant vertices and according to Lemma 1.1 every leaf (pendant) of $T$ belongs to the minimum restrained dominating set in $T$. Therefore, dominating set $A$ in $G$ can not be restrained. Thus, set $D$ is restrained dominating and is the minimum set with

$\gamma_{r t}(G)=|D|=$
$\sum_{i=0}^{\frac{r-1}{4}}\left(h^{r-4 i}+\right.$

$\left.h^{r-4 i-1}\right)=\frac{\left(h^{r+3}+h^{r+4}\right)\left(1-h^{-4\left(\left(\frac{r-1}{4}\right)+1\right)}\right)}{h^{4}-1}=(h+$ 1) $\left(\frac{h^{r+3}-1}{h^{4}-1}\right)$

(ii) if $r \equiv 2(\bmod 4)$ then set $D$ dominates the graph $G$, but in $G[V-D]$ root vertex $v_{0}$ is an isolated vertex, so $D \cup\left\{v_{0}\right\}$ is a total restrained dominating set in $G$ and it's the minimum. Therefore, in this case

$$
\begin{aligned}
& \gamma_{r t}(G)=1+\sum_{i=0}^{\left[\frac{r-1}{4}\right]}\left(h^{r-4 i}+h^{r-4 i-1}\right)=1+ \\
& \sum_{i=0}^{\frac{r-2}{4}}\left(h^{r-4 i}+h^{r-4 i-1}\right) \\
& =1+\frac{\left(h^{r+3}+h^{r+4}\right)\left(1-h^{-4\left(\frac{r-2}{4}+1\right)}\right)}{h^{4}-1}=1+\left(h^{2}+\right. \\
& h)\left(\frac{h^{r+2}-1}{h^{4}-1}\right)
\end{aligned}
$$


(iii) If $r \equiv 3(\bmod 4)$, then the set $D$ dominates $G$ except the root vertex. Let $D \cup\{u\}$ be the dominating set of $G$ where, $u$ is any vertex of depth one. So, $D \cup\{u\}$ is a minimum total restrained dominating set in $G$. Therefore the result is gotten.

$$
\begin{aligned}
& \gamma_{r t}(G)=1+\sum_{i=0}^{\left[\frac{r-1}{4}\right\rfloor}\left(h^{r-4 i}+h^{r-4 i-1}\right)=1+ \\
& \left(h^{r+3}+h^{r+4}\right) \frac{\left(1-h^{-4\left(\frac{r-3}{4}+1\right)}\right)}{h^{4}-1} \\
& =1+\left(h^{3}+h^{2}\right)\left(\frac{h^{r+1}-1}{h^{4}-1}\right)
\end{aligned}
$$

(iv) If $r \equiv 0(\bmod 4)$, then the set $D$ is the minimum total restrained dominating set in $G$ except the vertices of depth two and one plus the root vertex. Thus, $\gamma_{r t}(G)=1+h+2 h+$ $\sum_{i=0}^{\left\lfloor\frac{r-1}{4}\right\rfloor}\left(h^{r-4 i}+h^{r-4 i-1}\right)=1+h+2 h+\left(h^{4}+\right.$ $\left.h^{3}\right)\left(\frac{h^{r}-1}{h^{4}-1}\right)$.

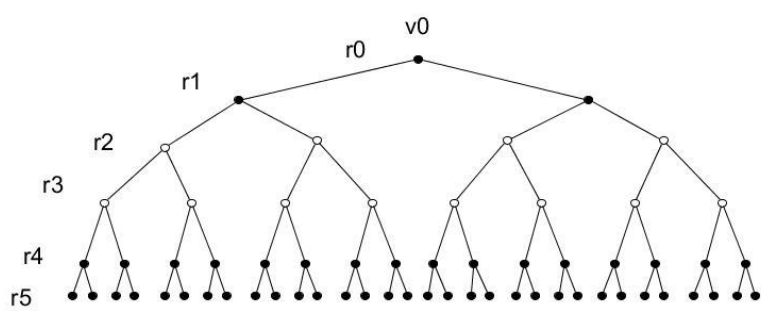

Figure 3: Total restrained dominating set in $T_{c, 2,5}$

\section{Secure domination in a complete $h$-ary tree}

Theorem 5 If $G=T_{c, h, r}$ is a complete $h$-ary tree; then for $r \geq 3$,

$$
\gamma_{S}(G)=\left\lfloor\frac{h^{r+1}-1}{h^{2}-1}\right\rfloor+h^{r-1}(h-1) .
$$

\section{Proof.}

Case1. If $r$ is odd.

Let's consider $D=A_{1} \cup A_{2}$, where $A_{1}=\bigcup_{i=0}^{\left\lfloor\frac{r}{2}\right\rfloor} D_{i}$ where $D_{i}=\{v: v$ is a vertex of depth $r-2 i-1\}$ in
$G \quad, \quad\left|A_{1}\right|=\sum_{i=0}^{\frac{r-1}{2}} h^{r-2 i-1}$, and $A_{2}=\{v: v$ is a vertex of depth $r\}$, such that $\left|A_{2}\right|=h^{r}-h^{r-1},|D|=\left|A_{1}\right|+\left|A_{2}\right|$ $=\sum_{i=0}^{\frac{r-1}{2}} h^{2 i}+h^{r}-h^{r-1}=\frac{h^{r+1}-1}{h^{2}-1}+h^{r-1}(h-1)$. Thus, all vertices of $G$ are dominated by set $D$.

Every vertex in $D_{i}$ is adjacent to some vertices in row $r_{i+1}$. Therefore, for each vertex $v$ in $D_{i}$ there is a vertex $u$ in $V-D$ such that, the swap ( $(D-$ $\{v\}) \cup\{u\})$ is a domination set since the vertices in $r_{i+1}$ are dominated by the vertices of $D_{i}$ in row $i$ and the vertices of $D_{i+2}$ in row $r_{i+2}$. Therefore, $D$ is the secure dominating set in $G$. Thus, $\gamma_{S}(G)=$ $\frac{h^{r+1}-1}{h^{2}-1}+h^{r-1}(h-1)$. If there is another dominating set say $F$, and $|F|<|D|$, then there are at least $h$ vertices of $G$ which are not dominated by set $F$. Thus, $D$ is the minimum and it is a secure dominating set. So that, the secure domination number in this case of $T_{c, h, r}$ is

$$
\begin{aligned}
\gamma_{S}(G)=|D|= & \frac{h^{r+1}-1}{h^{2}-1}+h^{r-1}(h-1) \\
& =\left\lfloor\frac{h^{r+1}-1}{h^{2}-1}\right\rfloor+h^{r-1}(h-1) .
\end{aligned}
$$

Case 2. If $r$ is even, then $=A_{1} \cup A_{2}$, where $D$ is a dominating set such that $A_{1}=\cup_{i=0}^{\left[\frac{r-1}{2}\right]} D_{i}$ where $D_{i}=\{v: v$ is a vertex of depth $r-2 i\}$ in $G$, and $\left|A_{1}\right|=\sum_{i=0}^{\left\lfloor\frac{r-1}{2}\right\rfloor} h^{r-2 i-1}$. While,

$A_{2}$ is the same set in Case 1.

Thus, $|D|=\left|A_{1}\right|+\left|A_{2}\right|$, and $D$ is the minimum secure dominating set as same proof in Case 1(Fig.4).

$$
\begin{aligned}
& \gamma_{S}(G)=|D|=\sum_{i=0}^{\left\lfloor\frac{r-1}{2}\right\rfloor} h^{r-2 i-1}+h^{r}-h^{r-1}= \\
& \frac{h^{r+1}-1}{h^{2}-1}+h^{r-1}(h-1)=\left\lfloor\frac{h^{r+1}-1}{h^{2}-1}\right\rfloor+h^{r-1}(h-1) .
\end{aligned}
$$




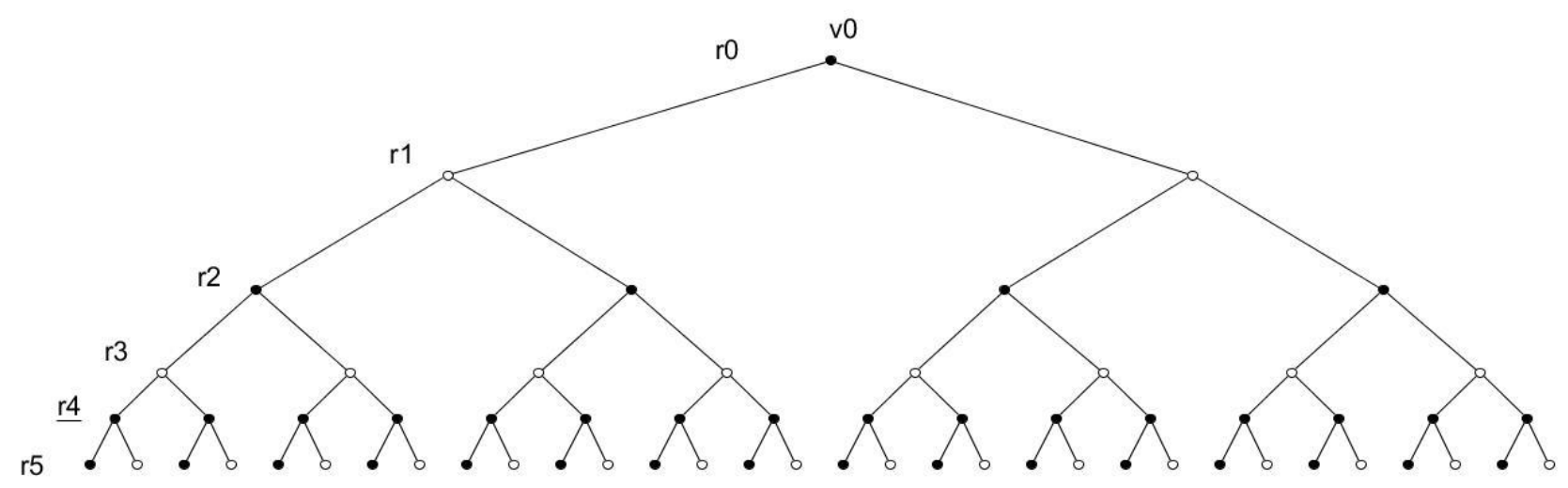

Figure 4. Secure dominating set in $T_{c, 2,5}$

\section{Conclusion:}

Domination number for some types of graph domination is calculated for a complete $h$-ary root tree $T_{c, h, r}$. A restrained domination number for complete $h$-ary root, $h \geq 2$, can be determined form $r \geq 3$, while the total restrained domination can be determined from $r \geq 5$. When, $r=3$ and 4 the complete $h$-ary tree has no total restrained domination. The independence restrained domination number equals to restrained domination number for the complete $h$-ary tree, when $r \equiv$ $0(\bmod 3)$. When $(r \equiv 1,2(\bmod 3)$, the graph has no independence restrained dominating set. The secure domination in a complete $h$-ary tree can be determined from $r \geq 3$.

\section{Authors' declaration:}

- Conflicts of Interest: None.

- We hereby confirm that all the Figures and Tables in the manuscript are mine ours. Besides, the Figures and images, which are not mine ours, have been given the permission for republication attached with the manuscript.

- Ethical Clearance: The project was approved by the local ethical committee in University of Technology.

\section{References:}

1. Claude B. The theory of graphs and its applications. London: Methuen. New York: Wiley.1962.

2. Frank H. Graph theory. London: Addison-Wesley. 1969.

3. Oystein O. Theory of graphs. Providence. RI: American Mathematical Society. 1962.

4. Teresa WH, Stephen TH, Peter JS. Fundamentals of domination in graphs. New York: Marcel Dekkar. 1998.
5. Hedetneimi ST, Laskar R .Topics in domination in graphs. Discrete Math. 1990;88.

6. Al-Harere M N, Khuda Bakhash PA. Tadpole domination in graphs. Baghdad Sci. J. 2018; 15(4):466-471.

7. Al-Harere MN, Omran AA . Binary operation in graphs. Bol. Soc. Paran. Mat. (2020); 38 (7): 59-67.

8. Al-Harere MN, Abdlhusein MA. Pitchfork domination in graphs. Discrete Mathematics, Algorithm and Applications.2020; 12(2): 2050025.

9. Al-Harere MN, Breesam AT. Further results on bidomination in graphs. AIP Conference Proceedings . 2019; 2096(1): 020013-1-020013-9.

10. Abdlhusein MA, Al-Harere MN. Pitchfork Domination and It's Inverse for Corona and Join Operations in Graphs .PIMS .2020; 1 (2): 51 - 55.

11. Abdlhusein MA, Al-Harere MN. Pitchfork Domination and It's Inverse for Complement graphs. Proceedings of IAM. 2020; 9(1):13-17.

12. Omran AA, Al Hwaeer $\mathrm{H}$ J. Modern roman domination in graphs .BJS (A). 2018; 36 (1): 45-54.

13. Jabour AA, Omran AA . Domination in discrete topology graph. AIP Conference Proceedings..2019; 2183(6): 030006.

14. Omran AA, Haneen H O. Hn domination in graphs. Baghdad Sci. J. 2019; 16(1):242-247.

15. Domke GS, Hattingh JH, Henning MA, Markus LR. Restrained domination in tress. Discrete Math. . 2000; 211(3): 1-9.

16. Nicanor T, Sergio R, Canoy J. Independent restrained domination in graphs. Appl. Math. Sci. 2014; 8 (121): $6033-6038$.

17. Hattingh JH, Jonck E, Joubert EJ, Plummer AR . Total restrained domination in trees. Discrete Math. 2007; 307: 1643-1650.

18. Houcine BM, Mustapha.C. On secure domination in graphs, Information Processing. Letters.2015; 115(10):786-790.

19. Burger AP, de Villiers A, van Vuuren JH.On Minimum secure dominating sets of graphs. QUAEST MATH. 2016; 39(2):189-202. 


\section{انواع مختلفة من الهيمنة في بيان شجرة شعاع الجذر المتكامل}

فاطمة احمد صادق

$$
\text { رشا جلال متلف }
$$

$$
\text { منال ناجي الحريري }
$$

قسم العلوم التطبيقية ، الجامعة التكنولوجية ، بغداد ، العراق

يعتبر البيان

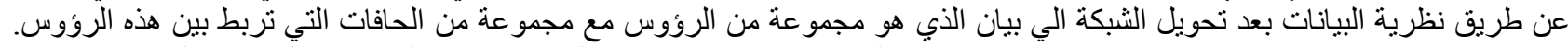

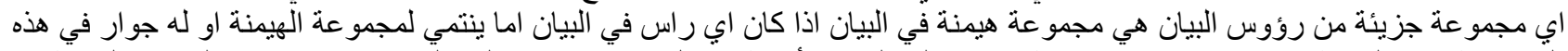

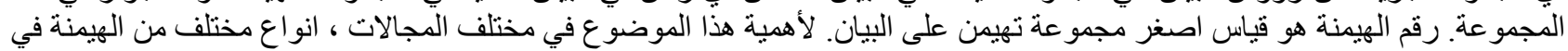

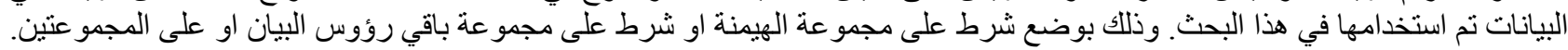

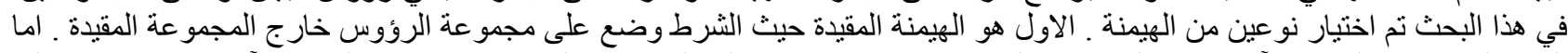

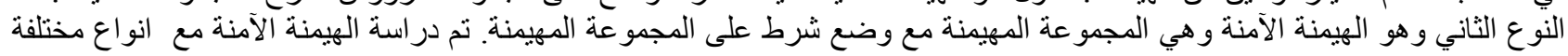

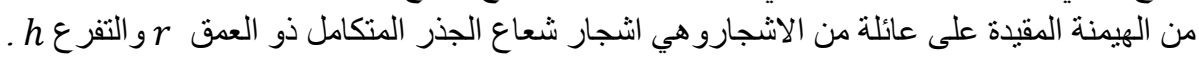

الكلمات المفتاحية : شجرة شعاع الجذر المتكامل ذو العمق r و التفرع h ، الهيمنة ، الهيمنة المقيدة ، الهيمنة الآمنة . 